\title{
CONICS AND CUBICS CONNECTED WITH A PLANE CUBIC BY CERTAIN COVARIANT RELATIONS*
}

BY

\section{HENRY S. WHITE}

It is to be expected that the systematic introduction of irrational covariants will enrich geometry with curves and surfaces, not previously observed or discussed, allied to any given fundamental system by projective relations. Well known irrationalities also must be expected often to reappear. In $1888 \mathrm{Dr}$. HILBERT $\uparrow$ remarked the existence of two nets of conics covariantly related to a general curve of third order in a plane. Two additional systems, nets of curves of the second class, can easily be defined by an equation closely analogous to Hilbert's. These systems of conics and the four cubics whose polars they are prove to be not entirely unknown hitherto, for their dually equivalent loci and envelopes form the basis of the one-to-one correspondences upon the point-cubic and the line-cubic respectively. That only two of every three such correspondences are found here is due to the domain of rationality that is assumed. By employing the irrationality that occurs in Hesse's canonical form of the cubic I am able to identify Hilbert's two systems of irrational covariant conics and to exhibit their relation to the other two systems just mentioned. As a consequence it is found possible to give explicitly covariant equations of definition for the two cubics which have the same Hessian and for those which have the same Cayleyan as a given fundamental cubic. These results are here derived by the aid of a canonical form of the cubic containing Hesse's irrationality. The desirable invariantive proofs of these results are given by Professor GoRdan in a paper presented by him to the American Mathematical Society for publication in its Transactions. $f$

\section{§1. Hilbert's covariant conics.}

The covariant of second degree in the coefficients of a ternary cubic $a$ or $a_{x}^{3}$ is written symbolically

$$
\left(a a^{\prime} u\right)^{2} a_{x} a_{x}^{\prime}
$$

* Presented to the Society at the Columbus meeting, Aug. 25, 1899, under a slightly different title. Received for publication Oct. 10, 1899.

† Lettre adressée à M. Hermite, Liouville's Journal, vol. 4, ser. 4, 1888.

$\ddagger P .9$ of this number.

Trans. Am. Math. Soc., 1. 
If this is equated to zero it denotes in variables $x_{1}, x_{2}, x_{3}$, the poloconic of the line

$$
u_{1} x_{1}+u_{2} x_{2}+u_{3} x_{3}=0 .
$$

Or if $u_{1}, u_{2}, u_{3}$ be taken as variables it is the tangential equation of the conic polar of a point $(x)$. If now the variables $(u)$ are replaced by symbols of any ternary $n$-ic $A_{x}^{n}$, we shall say that the equation

$$
\left(a a^{\prime} A\right)^{2} a_{x} a_{x}^{\prime} A_{x}^{n-2}=0
$$

denotes the poloconic, polocubic, or polo-n-ic of the curve $A_{x}^{n}=0$ with respect to the cubic $a_{x}^{3}=0$. HILBERT proposes to determine those conics, if any, which coincide with their poloconics. This requires the determination of a value $\lambda$ such that

$$
\left(a a^{\prime} A\right)^{2} a_{x} a_{x}^{\prime} \equiv \lambda \cdot A_{x}^{2} \text {. }
$$

It is found that the six values of $\lambda$ resulting from elimination of the unknown $A$ 's coincide by threes. Hence to the two values .

$$
\lambda= \pm \sqrt{\frac{1}{6} S}= \pm \sqrt{\frac{1}{6}\left(a a^{\prime} a^{\prime \prime}\right)\left(a a^{\prime} a^{\prime \prime \prime}\right)\left(a a^{\prime \prime} a^{\prime \prime \prime}\right)\left(a^{\prime} a^{\prime \prime} a^{\prime \prime \prime}\right)}
$$

there correspond two quadric forms each containing linearly three arbitrary parameters. So much Hir.bert states. In order to recognize these autopoloconics as known systems it will be convenient to use a canonical form of the fundamental cubic, due to Hesse.*

Referred to an inflexional triangle, the equation of the cubic takes the form

$$
a_{x}^{3}=x_{1}^{3}+x_{2}^{3}+x_{3}^{3}+6 m x_{1} x_{2} x_{3}=0 .
$$

All conic polars accordingly have the form :

$$
a_{y} a_{x}^{2}=\left(y_{1} x_{1}^{2}+y_{2} x_{2}^{2}+y_{3} x_{3}^{2}\right)+2 m\left(y_{1} x_{2} x_{3}+y_{2} x_{3} x_{1}+y_{3} x_{1} x_{2}\right)=0 .
$$

With respect to this triangle, the quantity $m$ may be termed indifferently the parameter of the cubic or the parameter of any one of its conic polars. The defining equation of the autopoloconics may be written with the aid of a bordered Hessian, as follows :

$$
2\left|\begin{array}{rrrl}
x_{1} & m x_{3} & m x_{2} & A_{1} \\
m x_{3} & x_{2} & m x_{1} & A_{2} \\
m x_{2} & m x_{1} & x_{3} & A_{3} \\
A_{1} & A_{2} & A_{3} & 0
\end{array}\right| \equiv \lambda A_{x}^{2} .
$$

Expanded, this identity gives three pairs of equations of condition like the following,

*Crelle's Journal, vol. 28, pp. 68-96, 1844. 


$$
\left\{\begin{array}{l}
2 m^{2} A_{11}+4 m A_{23}=\quad \lambda A_{11} \\
2 A_{11}+4 m^{2} A_{23}=-2 \lambda A_{23}{ }^{\circ}
\end{array}\right\}
$$

Eliminate $\lambda$ and write

$$
\frac{A_{23}}{A_{11}}=A=\frac{A_{31}}{A_{22}}=\frac{A_{12}}{A_{33}}
$$

There results the simple relation between parameters $m$ and $A$ :

$$
4 m A(m+A)+1=0 .
$$

This yields two values for $A$, which we shall distinguish as $A$ and $B$. The corresponding systems or nets of conics are these :

$$
\left\{\begin{array}{l}
\left(y_{1} x_{1}^{2}+y_{2} x_{2}^{2}+y_{3} x_{3}^{2}\right)+2 A\left(y_{1} x_{2} x_{3}+y_{2} x_{3} x_{1}+y_{3} x_{1} x_{2}\right)=0 \\
\left(y_{1} x_{1}^{2}+y_{2} x_{2}^{2}+y_{3} x_{3}^{2}\right)+2 B\left(y_{1} x_{2} x_{3}+y_{2} x_{3} x_{1}+y_{3} x_{1} x_{2}\right)=0
\end{array}\right\}
$$

the $y$ 's denoting arbitrary quantities. Notice now that these are the nets of conic polars belonging to the two cubics, which we will denote by corresponding symbols :

$$
\left\{\begin{array}{l}
A_{x}^{3}=x_{1}^{3}+x_{2}^{3}+x_{3}^{3}+6 A x_{1} x_{2} x_{3}=0 \\
B_{x}^{3}=x_{1}^{3}+x_{2}^{3}+x_{3}^{3}+6 B x_{1} x_{2} x_{3}=0
\end{array}\right\}
$$

These two cubics, from the form of their equations, are seen to belong to the syzygetic sheaf determined by the fundamental cubic $a$ and its Hessian. We have then this theorem:

To each general cubic curve there correspond two other cubics in its syzygetic sheaf, the conic polars of the latter being all autopoloconics of the former.

Further, since equation (7) is symmetric in the two parameters $m$ and $A$, we may add :

With respect to each of the latter two cubics the conic polars of the former constitute one net of autopoloconics.

But the two derivative cubics stand to each other in the same reciprocal relation; for from the conditions (7),

$$
4 m A(m+A)+1=0,4 m B(m+B)+1=0, A \neq B,
$$

there follows the relation

$$
4 A B(A+B)+1=0 .
$$

Hence we have the theorem :

These three cubics, the fundamental $a$ and the two derived cubics $A$ and $B$, constitute a closed system, the autopoloconics of each cubic being the conic polars of each of the other two cubics. 
Only one more step is needful for the complete recognition of this set of three. Modify the relation (7) between parameters by the factor $(m-A) / m A$; in the result,

$$
4\left(m^{2}-A^{2}\right)+\left(\frac{1}{A}-\frac{1}{m}\right)=0,
$$

the parameters can be separated, so that by use of $(10)$ we have the equality

$$
-\frac{4 m^{3}-1}{6 m}=-\frac{4 A^{3}-1}{6 A}=-\frac{4 B^{3}-1}{6 B}=c .
$$

This rational function having the same value for all three parameters is seen on inspection to be the parameter of the Cayleyan curve of third class, (the envelope of lines constituting degenerate conic polars in each net). Since every non-singular cubic class-curve is Cayleyan to three point-cubies, the relation may: be re-stated thus:

The three cubics which have a Cayleyan in common are characterized fully by the property that all conic polars of one (and therefore of each one) are autopoloconics of both the others.

\section{§2. Two covariant systems of curves of second class.}

The mixed covariant $\Theta=\left(a a^{\prime} u\right)^{2} a_{x} a_{x}^{\prime}$, used above as a transformer for forms in variables $(x)$, may be used equally well to transform those with variables $(u)$. The curve $u_{a}^{n}=0$ is transformed into the curve $\left(a a^{\prime} u\right)^{2} a_{a} a_{a}^{\prime} u_{a}^{n-2}=0$. If the curve is transformed thus into itself, let it be termed an autopolar of the fundamental cubic; and there will arise the problem, to determine conic autopolars, cubic autopolars, etc. A conic autopolar will have as defining equation

$$
\left(a a^{\prime} u\right)^{2} a_{a} a_{a}^{\prime} \equiv \mu \cdot u_{a}^{\prime},
$$

or

$$
2\left|\begin{array}{rrrr}
\alpha_{1} & m \alpha_{3} & m \alpha_{2} & u_{1} \\
m \alpha_{3} & \alpha_{2} & m \alpha_{1} & u_{2} \\
m \alpha_{2} & m \alpha_{1} & \alpha_{3} & u_{3} \\
u_{1} & u_{2} & u_{3} & 0
\end{array}\right| \equiv \mu \cdot u_{a}^{2} .
$$

The six conditions of this identity fall into three pairs as before and determine two discrete $\infty^{2}$ webs of conics. From one pair of conditions :

$$
\left\{\begin{array}{l}
2 m^{2} \alpha_{11}-2 \alpha_{23}=\mu \alpha_{11} \\
4 m \alpha_{11}-4 m^{2} \alpha_{23}=2 \mu \alpha_{23}
\end{array}\right\}
$$

eliminate $\mu$, set $\alpha_{23} / \alpha_{11}=\alpha$, and call the roots of the resulting quadric $\alpha$ and $\beta$, i. e.,

$$
\left\{\begin{array}{l}
\alpha^{2}-2 m^{2} \alpha+m=0 \\
\beta^{2}-2 m^{2} \beta+m=0
\end{array}\right\}
$$


The two systems of conics and the cubics whose polar systems they are respectively have the equations

$$
\left\{\begin{array}{l}
\left(v_{1} u_{1}^{2}+v_{2} u_{2}^{2}+v_{3} u_{3}^{2}\right)+2 \alpha \cdot\left(v_{1} u_{2} u_{3}+v_{2} u_{3} u_{1}+v_{3} u_{1} u_{2}\right)=0, \\
\left(v_{1} u_{1}^{2}+v_{2} u_{2}^{2}+v_{3} u_{3}^{2}\right)+2 \beta \cdot\left(v_{1} u_{2} u_{3}+v_{2} u_{3} u_{1}+v_{3} u_{1} u_{2}\right)=0,
\end{array}\right\}
$$

the $v$ 's being arbitrary quantities, and

$$
\left\{\begin{array}{l}
u_{a}^{3}=u_{1}^{3}+u_{2}^{3}+u_{3}^{3}+6 \alpha u_{1} u_{2} u_{3}=0, \\
u_{\beta}^{3}=u_{1}^{3}+u_{2}^{3}+u_{3}^{3}+6 \beta u_{1} u_{2} u_{3}=0 .
\end{array}\right\}
$$

From (14) we find the relation between $\alpha$ and $\beta$ to be

$$
2 \alpha^{2} \beta^{2}-(\alpha+\beta)=0 \text {. }
$$

If $\alpha$ is considered constant, $\beta$ and $\gamma$ may denote roots of this equation, and the relation between them is found to be of the same form :

$$
2 \beta^{2} \gamma^{2}-(\beta+\gamma)=0 \text {. }
$$

These three again constitute a closed set. The parameters can be separated, giving the equal rational functions :

$$
-\frac{1+2 \alpha^{3}}{6 \alpha^{2}}=-\frac{1+2 \beta^{3}}{6 \beta^{2}}=-\frac{1+2 \gamma^{3}}{6 \gamma^{2}}=C^{\prime},
$$

and this is the parameter of the Hessian* of each of the cubics (16). Notice also that the unsymmetric relation (14) becomes the symmetric $(17 a)$ if we take $\gamma=-1 / 2 m$.

The two systems of conic autopolars of a plane cubic are the conic polars of two curves of third class which have a common Hessian; and this is also the Hessian of the curve of third class which is "conjugate" or doubly apolar to the fundamental curve of third order.

The term conjugate, introduced by Hesse (l. c.), has been applied more recently to curves which are simply apolar. The curves here considered have all conic polars of one apolar to all conic polars of the other ; hence the term doubly apolar seems to me preferable. It is easily verified that any polar of the one,

$$
u_{1}^{2}-\frac{2}{2 m} u_{2} u_{3}=0 \text {, }
$$

is apolar to three independent conic polars of the other,

$$
\begin{aligned}
& x_{1}^{2}+2 m x_{2} x_{3}=0, \\
& x_{2}^{2}+2 m x_{3} x_{1}=0, \\
& x_{3}^{2}+2 m x_{1} x_{2}=0 .
\end{aligned}
$$

* See Salmon, Higher Plane Curves (3d ed.), p. 190. 
The two webs of conic autopolars are connected in this same manner with the two nets of autopoloconics : that is to say, each web is apolar to one of the nets, and is autopolar to the cubic of the other net. This is already evident geometrically from the fact that the Cayleyan of any cubic is the Hessian of its doubly apolar cubic.* Otherwise the mutual apolarity may be readily verified in the following manner from the characteristic equations of conics in the net and web respectively. Let $A_{x}^{2}$ and $u_{a}^{2}$ satisfy relations (2) and (12):

$$
\begin{aligned}
& \left(a a^{\prime} A\right)^{2} a_{x} a_{x}^{\prime} \equiv \lambda_{A} \cdot A_{x}^{2}, \\
& \left(a a^{\prime} u\right)^{2} a_{a} a_{a}^{\prime} \equiv \mu_{a} \cdot u_{a}^{2},
\end{aligned}
$$

and let $\mu_{a}$ denote the value of $\mu$ different from $\lambda_{A}$. Then operating on both members of these identities with

we shall find

$$
\sum_{i, k}\left(\alpha_{i k} \frac{\partial^{2}}{\partial x_{i} \partial x_{k}}\right), \quad \sum_{i, k}\left(A_{i k}-\frac{\partial^{2}}{\partial u_{i} \partial u_{k}}\right)
$$

$$
\begin{aligned}
& \left(a a^{\prime} A\right)^{2} a_{a} a_{a}^{\prime} \equiv \lambda_{d} \cdot A_{a}^{2}, \\
& \left(a a^{\prime} A\right)^{2} a_{a} a_{a}^{\prime} \equiv \mu_{a} \cdot A_{a}^{2} ;
\end{aligned}
$$

or since $\lambda_{\Delta} \neq \mu_{a}$ (indeed $\mu_{a}=-\lambda_{\Delta}$ ), we have by subtraction :

$$
A_{a}^{2}=0 \text {. Similarly } B_{\beta}^{2}=0 \text {. }
$$

Remembering that these conics are polars of cubics, we may state the relations of the cubics as follows :

The two point cubics $A_{x}^{3}=0$ and $B_{x}^{3}=0$ satisfying the conditions for autopoloconics

$$
\begin{aligned}
\left(a a^{\prime} A\right)^{2} a_{x} a_{x}^{\prime} A_{y} & \equiv \lambda \cdot A_{x}^{2} A_{y}, \\
\left(a a^{\prime} B\right)^{2} a_{x} a_{x}^{\prime} B_{y} & \equiv-\lambda \cdot B_{x}^{2} B_{y},
\end{aligned}
$$

are respectively doubly apolar to the two line cubics $u_{\alpha}^{3}=0$ and $u_{\beta}^{3}=0$ which satisfy the conditions for conic autopolars

$$
\begin{aligned}
& \left(a a^{\prime} u\right)^{2} a_{a} a_{\alpha}^{\prime} v_{\alpha} \equiv-\lambda \cdot u_{a}^{2} v_{\alpha}, \\
& \left(a a^{\prime} u\right)^{2} a_{\beta} a_{\beta}^{\prime} v_{\beta} \equiv \lambda \cdot u_{\beta}^{2} v_{\beta} .
\end{aligned}
$$

In other words, the two equations subsist identically :

$$
\begin{aligned}
A_{\alpha}^{2} A_{x} u_{\alpha} & \equiv 0, \\
B_{\beta}^{2} B_{x} u_{\beta} & \equiv 0 .
\end{aligned}
$$

Moreover the conic polars of the curves $A, B, \alpha$, and $\beta$ are autopoloconics respectively to the curves $B, A, \beta$, and $\alpha$, and conic autopolars respectively to the curves

* Clebsch-Lindemann, Vorlesungen über Geometrie, vol. I., p. 521. 
$\beta, a, B$ and $A$. The conic polars of the fundamental cubic a are autopoloconics of $A$ and $B$, and conic auto ${ }_{1}$, lars to the curves $\alpha$ and $\beta$. That is to say, the following characteristic relations are fulfilled:

$$
\begin{aligned}
& \left(A A^{\prime} B\right)^{2} A_{x} A_{x}^{\prime} B_{y} \equiv \pi \cdot B_{x}^{2} B_{y}, \\
& \left(B B^{\prime} A\right)^{2} B_{x} B_{x}^{\prime} A_{y} \equiv \rho \cdot A_{x}^{2} A_{y}, \\
& \left(\alpha \alpha^{\prime} \beta\right)^{2} u_{a} u_{\alpha^{\prime}} v_{\beta} \equiv \sigma \cdot u_{\beta}^{2} v_{\beta}, \\
& \left(\beta \beta^{\prime} \alpha\right)^{2} u_{\beta} u_{\beta^{\prime}} v_{\alpha} \equiv \tau \cdot u_{\alpha}^{2} v_{a} \\
& \left(A A^{\prime} u\right)^{2} A_{\beta} A_{\beta}^{\prime} v_{\beta} \equiv-\pi \cdot u_{\beta}^{2} v_{\beta}, \\
& \left(B B^{\prime} u\right)^{2} B_{a} B_{a}^{\prime} v_{\alpha} \equiv-\rho \cdot u_{a}^{2} v_{a}, \\
& \left(\alpha \alpha^{\prime} x\right)^{2} B_{a} B_{a^{\prime}} B_{y} \equiv-\sigma \cdot B_{x}^{2} B_{y}, \\
& \left(\beta \beta^{\prime} x\right)^{2} A_{\beta} A_{\beta^{\prime}} A_{y} \equiv-\tau \cdot A_{x}^{2} A_{y} .
\end{aligned}
$$

§3. Defining equations for two order-cubics and two class-cubics related to the Hessian.

Denote by $f=a_{x}^{3}=0$ the fundamental cubic, and by $\pi=u_{\pi}^{3}=0$ its doubly apolar curve of third class ; we may then speak of the curve $f$ and the curve $\pi$. It is known that an interchange of the curves $f$ and $\pi$ interchanges also the Hessian and the Cayleyan, the point-coördinates and line-coördinates, etc. Accordingly by forming the covariant $\Theta$ for $\pi$ instead of for $f$, we shall be able to define algebraically as irrational covariants those systems of conics and their cubics which are related to the Hessian just as those already discussed were related to the Cayleyan. For this purpose, take from Clebsch and Gordan's admirable paper in Mathematische Annalen, vol. 6, two formulæ,

$$
\begin{gathered}
\pi=u_{\pi}^{3}=S \mathrm{~T}-T \Sigma, \\
\Theta^{(\pi)}=\left(\pi \pi^{\prime} x\right)^{2} u_{\pi^{\prime}} u_{\pi^{\prime}}=R\left(\mathrm{~K}-\frac{1}{6} S \Theta\right),
\end{gathered}
$$

(particularized from (91), p. 494), where $\mathrm{K}$ denotes the covariant $\Theta$ formed for the Hessian $\Delta_{x}^{3}$ instead of the curve $f$ or $a_{x}^{3}$, viz.,

$$
\mathbf{K}=\left(\Delta \Delta^{\prime} u\right)^{2} \Delta_{x} \Delta_{x}^{\prime},
$$

and $R$ denotes the discriminant of the cubic,

$$
R=T^{2}-\frac{1}{6} S^{3}
$$

By omitting the factor $R$, as our cubic is assumed to be non-singular, these changes give us the theorem : 
The two point-cubics having the same Hessian with a given fundamental cubic $a_{x}^{3}=0$ being denoted by equations $F_{x}^{3}=0$ and $G_{x}^{3}=0$; and the two line-cubics respectively doubly apolar to these, having therefore this Hessian as their common Cayleyan curve, being denoted by equations $u_{\phi}^{3}=0$ and $u_{\psi}^{3}=0$, these two pairs of curves are completely defined by the covariant equations:

$$
\begin{aligned}
& \left(\Delta \Delta^{\prime} F^{\prime}\right)^{2} F_{y} \Delta_{x} \Delta_{x}^{\prime}-\frac{1}{6} S\left(a a^{\prime} F^{\prime}\right)^{2} F_{y} a_{x} a_{x}^{\prime} \equiv \mu \cdot F_{x}^{2} F_{y}, \\
& \left(\Delta \Delta^{\prime} G\right)^{2} G_{y} \Delta_{x} \Delta_{x}^{\prime}-\frac{1}{6} S\left(a a^{\prime} G^{2} G_{y} a_{x} a_{x}^{\prime} \equiv-\mu \cdot G_{x}^{2} G_{y},\right. \\
& \left(\Delta \Delta^{\prime} u\right)^{2} v_{\phi} \Delta_{\phi} \Delta_{\phi}^{\prime}-\frac{1}{6} S\left(a a^{\prime} u\right)^{2} v_{\phi} a_{\phi} a_{\phi}^{\prime} \equiv-\mu \cdot u_{\phi}^{2} v_{\phi}, \\
& \left(\Delta \Delta^{\prime} u\right)^{2} v_{\psi} \Delta_{\psi} \Delta_{\psi}^{\prime}-\frac{1}{6} S\left(a a^{\prime} u\right)^{2} v_{\psi} a_{\psi} a_{\psi}^{\prime} \equiv \mu \cdot u_{\psi}^{2} v_{\psi} .
\end{aligned}
$$

where $\mu$ is a constant determinable from the equations.

To these might be appended also the sets analogous to those in (20), but the geometric relations are already sufficiently evident.

NORTH WESTERN UNIVERSITY. 